\title{
Elliptic Hypergeometric Solutions to Elliptic Difference Equations ${ }^{\star}$
}

\author{
Alphonse P. MAGNUS
}

Université catholique de Louvain, Institut mathématique, 2 Chemin du Cyclotron, B-1348 Louvain-La-Neuve, Belgium

E-mail: alphonse.magnus@uclouvain.be

URL: http://perso.uclouvain.be/alphonse.magnus/

Received December 01, 2008, in final form March 20, 2009; Published online March 27, 2009 doi:10.3842/SIGMA.2009.038

\begin{abstract}
It is shown how to define difference equations on particular lattices $\left\{x_{n}\right\}$, $n \in \mathbb{Z}$, made of values of an elliptic function at a sequence of arguments in arithmetic progression (elliptic lattice). Solutions to special difference equations have remarkable simple interpolatory expansions. Only linear difference equations of first order are considered here.
\end{abstract}

Key words: elliptic difference equations; elliptic hypergeometric expansions

2000 Mathematics Subject Classification: 39A70; 41A20

Nacht und Stürme werden Licht

Choral Fantasy, Op. 80

\section{Difference equations on elliptic lattices}

\subsection{The difference operator}

We consider functional equations involving the difference operator

$$
(\mathcal{D} f)(x)=\frac{f(\psi(x))-f(\varphi(x))}{\psi(x)-\varphi(x)} .
$$

Most instances [26] are $(\varphi(x), \psi(x))=(x, x+h)$, or the more symmetric $(x-h / 2, x+h / 2)$, or also $(x, q x)$ in $q$-difference equations $[13,16,17]$. Recently, more complicated forms $(r(x)-$ $\sqrt{s(x)}, r(x)+\sqrt{s(x)})$ have appeared $[1,2,16,17,22,23,27,28,24]$, where $r$ and $s$ are rational functions.

This latter trend will be examined here: we need, for each $x$, two values $f(\varphi(x))$ and $f(\psi(x))$ for $f$. A first-order difference equation is

$$
\mathcal{F}(x, f(\varphi(x)), f(\psi(x)))=0, \quad \text { or } \quad f(\varphi(x))-f(\psi(x))=\mathcal{G}(x, f(\varphi(x)), f(\psi(x)))
$$

if we want to emphasize the difference of $f$. There is of course some freedom in this latter writing. Only symmetric forms in $\varphi$ and $\psi$ will be considered here:

$$
(\mathcal{D} f)(x)=\mathscr{F}(x, f(\varphi(x)), f(\psi(x))) \text {, }
$$

where $\mathcal{D}$ is the divided difference operator (1) and where $\mathscr{F}$ is a symmetric function of its two last arguments.

${ }^{\star}$ This paper is a contribution to the Proceedings of the Workshop "Elliptic Integrable Systems, Isomonodromy Problems, and Hypergeometric Functions" (July 21-25, 2008, MPIM, Bonn, Germany). The full collection is available at http://www.emis.de/journals/SIGMA/Elliptic-Integrable-Systems.html 
For instance, a linear difference equation of first order may be written as

$$
a(x) f(\varphi(x))+b(x) f(\psi(x))+c(x)=0,
$$

as well as

$$
\alpha(x)(\mathcal{D} f)(x)=\beta(x)[f(\varphi(x))+f(\psi(x))]+\gamma(x),
$$

with $\alpha(x)=[b(x)-a(x)][\psi(x)-\varphi(x)] / 2, \beta(x)=-[a(x)+b(x)] / 2$, and $\gamma(x)=-c(x)$.

The simplest choice for $\varphi$ and $\psi$ is to take the two determinations of an algebraic function of degree 2 , i.e., the two $y$-roots of

$$
F(x, y)=X_{0}(x)+X_{1}(x) y+X_{2}(x) y^{2}=0,
$$

where $X_{0}, X_{1}$, and $X_{2}$ are rational functions.

Note that the sum and the product of $\varphi$ and $\psi$ are the rational functions

$$
\varphi+\psi=-X_{1} / X_{2}, \quad \varphi \psi=X_{0} / X_{2} .
$$

\subsection{The corresponding lattice, or grid}

Difference equations must allow the recovery of $f$ on a whole set of points. An initial-value problem for a first order difference equation starts with a value for $f\left(y_{0}\right)$ at $x=x_{0}$, where $y_{0}$ is one root of (2a) at $x=x_{0}$. The difference equation at $x=x_{0}$ relates then $f\left(y_{0}\right)$ to $f\left(y_{1}\right)$, where $y_{1}$ is the second root of (2a) at $x_{0}$. We need $x_{1}$ such that $y_{1}$ is one of the two roots of (2a) at $x_{1}$, so for one of the roots of $F\left(x, y_{1}\right)=0$ which is not $x_{0}$. Here again, the simplest case is when $F$ is of degree 2 in $x$ :

$$
F(x, y)=Y_{0}(y)+Y_{1}(y) x+Y_{2}(y) x^{2}=0 .
$$

Both forms (2a) and (2c) hold simultaneously when $F$ is biquadratic:

$$
F(x, y)=\sum_{i=0}^{2} \sum_{j=0}^{2} c_{i, j} x^{i} y^{j} .
$$

The construction where successive points on the curve $F(x, y)=0$ are $\left(x_{n}, y_{n}\right),\left(x_{n}, y_{n+1}\right)$, $\left(x_{n+1}, y_{n+1}\right)$, is called "T-algorithm" in [34, Theorem 6], see also the Fritz John's algorithm in $[4,5,6]$. The sequence $\left\{x_{n}\right\}$ is then an instance of elliptic lattice, or grid.

Of course, the sequence $\left\{y_{n}\right\}$ is elliptic too, $x_{n}$ and $y_{n}$ have elliptic functions representations

$$
x_{n}=\mathcal{E}_{1}\left(t_{0}+n h\right), \quad y_{n}=\mathcal{E}_{2}\left(t_{0}+n h\right),
$$

where $\left(x=\mathcal{E}_{1}(t), y=\mathcal{E}_{2}(t)\right)$ is a parametric representation of the biquadratic curve $F(x, y)=0$ with the $F$ of (3).

Note that the names of the $x$ - and $y$-lattices are sometimes inverted, as in [34, equation (1.2)]

As $y_{n}$ and $y_{n+1}$ are the two roots in $t$ of $F\left(x_{n}, t\right)=X_{0}\left(x_{n}\right)+X_{1}\left(x_{n}\right) t+X_{2}\left(x_{n}\right) t^{2}=0$, useful identities are

$$
y_{n}+y_{n+1}=-\frac{X_{1}\left(x_{n}\right)}{X_{2}\left(x_{n}\right)}, \quad y_{n} y_{n+1}=\frac{X_{0}\left(x_{n}\right)}{X_{2}\left(x_{n}\right)},
$$

from $(2 b)$, and the direct formula

$$
y_{n} \text { and } y_{n+1}=\frac{-X_{1}\left(x_{n}\right) \pm \sqrt{P\left(x_{n}\right)}}{2 X_{2}\left(x_{n}\right)},
$$


where

$$
P=X_{1}^{2}-4 X_{0} X_{2}
$$

is a polynomial of degree 4 .

Also, as $x_{n+1}$ and $x_{n}$ are the two roots in $t$ of $F\left(t, y_{n+1}\right)=0$,

$$
x_{n}+x_{n+1}=-\frac{Y_{1}\left(y_{n+1}\right)}{Y_{2}\left(y_{n+1}\right)}, \quad x_{n} x_{n+1}=\frac{Y_{0}\left(y_{n+1}\right)}{Y_{2}\left(y_{n+1}\right)} .
$$

As the operators considered here are symmetric in $\varphi(x)$ and $\psi(x)$, we do not need to define precisely what $\varphi$ and $\psi$ are, i.e., we only need to know the pair $(\varphi, \psi)$, and not the ordered pair. However, once a starting point $\left(x_{0}, y_{0}\right)$ is chosen, it will be convenient to define $\varphi\left(x_{n}\right)=y_{n}$ and $\psi\left(x_{n}\right)=y_{n+1}, n \in \mathbb{Z}$.

Special cases. We already encountered the usual difference operators $(\varphi(x), \psi(x))=(x, x+h)$ or $(x-h, x)$ or $(x-h / 2, x+h / 2)$ corresponding to $X_{2}(x) \equiv 1, X_{1}$ of degree $1, X_{0}$ of degree 2 with $P=X_{1}^{2}-4 X_{0} X_{2}$ of degree 0 . For the geometric difference operator, $P$ is the square of a first degree polynomial. For the Askey-Wilson operator $[1,2,15,16,22,23], P$ is an arbitrary second degree polynomial.

The formulas for the sequences $x_{n}$ and $y_{n}$ are in these three special cases

$$
\begin{aligned}
& \left(x_{n}, y_{n}\right)=\left(x_{0}+n h, y_{0}+n h\right) ; \quad\left(a+b q^{n}, u+v q^{n}\right) \\
& \left(a+b q^{n}+c q^{-n}, u+v q^{n}+w q^{-n}\right) .
\end{aligned}
$$

\subsection{Difference of a rational function}

From $(2 \mathrm{~b})$, when the divided difference operator $\mathcal{D}$ of $(1)$ is applied to a rational function, the result is still a rational function.

The difference operator applied to a simple rational function is of special interest.

Let $f(x)=\frac{1}{x-A}$, then

$$
\begin{aligned}
\mathcal{D} \frac{1}{x-A} & =\frac{1}{\psi(x)-\varphi(x)}\left[\frac{1}{\psi(x)-A}-\frac{1}{\varphi(x)-A}\right]=-\frac{1}{(\psi(x)-A)(\varphi(x)-A)} \\
& =-\frac{X_{2}(x)}{X_{0}(x)+A X_{1}(x)+A^{2} X_{2}(x)},
\end{aligned}
$$

and let $\left\{\left(x_{n}^{\prime}, y_{n}^{\prime}\right),\left(x_{n}^{\prime}, y_{n+1}^{\prime}\right)\right\}$ be the elliptic sequence on the biquadratic curve $F(x, y)=0$ such that $y_{0}^{\prime}=A$, then

$$
\mathcal{D} \frac{1}{x-A}=-\frac{X_{2}(x)}{Y_{2}(A)\left(x-x_{0}^{\prime}\right)\left(x-x_{-1}^{\prime}\right)},
$$

as the denominator is $F(x, A)$, and the two $x$-roots of $F(x, A)=F\left(x, y_{0}^{\prime}\right)=0$ are $x_{0}^{\prime}$ and $x_{-1}^{\prime}$, from the opening discussion of Section 1.2.

The $\mathcal{D}$ operator applied to a general rational function yields a rational function with the factor $X_{2}$. It seems sometimes fit to define a difference operator as our $\mathcal{D}$ divided by $X_{2}$, as by V.P. Spiridonov and A.S. Zhedanov in Section 6 of [32]. See also Section 2 of [34].

A general rational function is generically a sum of simple rational functions of type (5), say, $1 /(x-A), 1 /(x-B)$, etc. The difference has poles at $x_{0}^{\prime}$ and $x_{-1}^{\prime}$, also at $x_{0}^{\prime \prime}$ and $x_{-1}^{\prime \prime}$ if $B=y_{0}^{\prime \prime}$, etc., so that the degree of $\mathcal{D} f$ is usually twice the degree of $f$. However, the difference of a rational function of denominator $\left(x-y_{0}^{\prime}\right)\left(x-y_{1}^{\prime}\right) \cdots\left(x-y_{n}^{\prime}\right), \mathcal{D} f$ has no other poles than $x_{-1}^{\prime}, x_{0}^{\prime}, \ldots, x_{n}^{\prime}$. This is also discussed in $[32,34]$. 
So, let $\left\{\left(x_{n}, y_{n}\right),\left(x_{n}, y_{n+1}\right)\right\}$ be a first elliptic sequence on the biquadratic curve $F(x, y)=0$, and $\left\{\left(x_{n}^{\prime}, y_{n}^{\prime}\right),\left(x_{n}^{\prime}, y_{n+1}^{\prime}\right)\right\}$ be another elliptic sequence on the same curve. The two sequences have the same formula (4), but with different starting values $t_{0}$ and $t_{0}^{\prime}$.

Now, let

$$
\mathcal{X}_{n}(x)=\frac{\left(x-x_{0}\right) \cdots\left(x-x_{n-1}\right)}{\left(x-x_{1}^{\prime}\right) \cdots\left(x-x_{n}^{\prime}\right)} \quad \text { and } \quad \mathcal{Y}_{n}(x)=\frac{\left(x-y_{0}\right) \cdots\left(x-y_{n-1}\right)}{\left(x-y_{1}^{\prime}\right) \cdots\left(x-y_{n}^{\prime}\right)}
$$

See that

$$
\mathcal{D} \mathcal{Y}_{n}(x)=C_{n} X_{2}(x) \frac{\mathcal{X}_{n-1}(x)}{\left(x-x_{0}^{\prime}\right)\left(x-x_{n}^{\prime}\right)} .
$$

Indeed, $\left(\varphi(x)-y_{0}\right)\left(\varphi(x)-y_{1}\right) \cdots\left(\varphi(x)-y_{n-1}\right)$ and $\left(\psi(x)-y_{0}\right)\left(\psi(x)-y_{1}\right) \cdots\left(\psi(x)-y_{n-1}\right)$ both vanish at $x=x_{0}, x_{1}, \ldots, x_{n-2} ;\left(\varphi(x)-y_{1}^{\prime}\right)\left(\varphi(x)-y_{2}^{\prime}\right) \cdots\left(\varphi(x)-y_{n}^{\prime}\right)$ vanishes at $x=x_{1}^{\prime}, \ldots, x_{n}^{\prime}$, whereas $\left(\psi(x)-y_{1}^{\prime}\right)\left(\psi(x)-y_{2}^{\prime}\right) \cdots\left(\psi(x)-y_{n}^{\prime}\right)$ vanishes at $x=x_{0}^{\prime}, \ldots, x_{n-1}^{\prime}$.

Simple fractions give

$$
\mathcal{D} \frac{1}{x-y_{k}^{\prime}}=-\frac{X_{2}(x)}{Y_{2}\left(y_{k}^{\prime}\right)\left(x-x_{k-1}^{\prime}\right)\left(x-x_{k}^{\prime}\right)}
$$

as seen earlier in (5).

The constant $C_{n}$ is found through particular values of $x$, either $x_{-1}$, where $\mathcal{Y}_{n}(\psi(x))=0$ but $\mathcal{Y}_{n}(\varphi(x)) \neq 0$, or $x_{n-1}$, where $\mathcal{Y}_{n}(\varphi(x))=0$ but $\mathcal{Y}_{n}(\psi(x)) \neq 0$ :

$$
\begin{aligned}
C_{n} & =-\frac{\mathcal{Y}_{n}\left(\varphi\left(x_{-1}\right)=y_{-1}\right)\left(x_{-1}-x_{0}^{\prime}\right)\left(x_{-1}-x_{n}^{\prime}\right)}{\left(y_{0}-y_{-1}\right) X_{2}\left(x_{-1}\right) \mathcal{X}_{n-1}\left(x_{-1}\right)}, \\
C_{n} & =\frac{\mathcal{Y}_{n}\left(\psi\left(x_{n-1}\right)=y_{n}\right)\left(x_{n-1}-x_{0}^{\prime}\right)\left(x_{n-1}-x_{n}^{\prime}\right)}{\left(y_{n}-y_{n-1}\right) X_{2}\left(x_{n-1}\right) \mathcal{X}_{n-1}\left(x_{n-1}\right)}
\end{aligned}
$$

(of course, $C_{0}=0$ ). Or through residues at $x_{0}^{\prime}$, where $\mathcal{Y}_{n}(\psi(x))=\infty$, or $x_{n}^{\prime}$ where $\mathcal{Y}_{n}(\varphi(x))=\infty$,

$$
\begin{aligned}
C_{n} & =\frac{\left(y_{1}^{\prime}-y_{0}\right) \cdots\left(y_{1}^{\prime}-y_{n-1}\right)}{\frac{d \psi\left(x_{0}^{\prime}\right)}{d x}\left(y_{1}^{\prime}-y_{2}^{\prime}\right) \cdots\left(y_{1}^{\prime}-y_{n}^{\prime}\right)} \frac{x_{0}^{\prime}-x_{n}^{\prime}}{\left(y_{1}^{\prime}-y_{0}^{\prime}\right) X_{2}\left(x_{0}^{\prime}\right) \mathcal{X}_{n-1}\left(x_{0}^{\prime}\right)}, \\
C_{n} & =-\frac{\left(y_{n}^{\prime}-y_{0}\right) \cdots\left(y_{n}^{\prime}-y_{n-1}\right)}{\left(y_{n}^{\prime}-y_{1}^{\prime}\right) \cdots\left(y_{n}^{\prime}-y_{n-1}^{\prime}\right) \frac{d \varphi\left(x_{n}^{\prime}\right)}{d x} \frac{x_{n}^{\prime}-x_{0}^{\prime}}{\left(y_{n+1}^{\prime}-y_{n}^{\prime}\right) X_{2}\left(x_{n}^{\prime}\right) \mathcal{X}_{n-1}\left(x_{n}^{\prime}\right)} .}
\end{aligned}
$$

We shall also need the operator $\mathcal{M}$ defined as

$$
(\mathcal{M} f)(x)=[f(\varphi(x))+f(\psi(x))] / 2,
$$

which sends rational functions to rational functions too, usually of double degree, but without particular factor.

With this operator $\mathcal{M}$,

$$
\begin{aligned}
2\left(\mathcal{M Y}_{n}\right)(x)= & \frac{\left(\varphi(x)-y_{0}\right)\left(\varphi(x)-y_{1}\right) \cdots\left(\varphi(x)-y_{n-1}\right)}{\left(\varphi(x)-y_{1}^{\prime}\right)\left(\varphi(x)-y_{2}^{\prime}\right) \cdots\left(\varphi(x)-y_{n}^{\prime}\right)} \\
& +\frac{\left(\psi(x)-y_{0}\right)\left(\psi(x)-y_{1}\right) \cdots\left(\psi(x)-y_{n-1}\right)}{\left(\psi(x)-y_{1}^{\prime}\right)\left(\psi(x)-y_{2}^{\prime}\right) \cdots\left(\psi(x)-y_{n}^{\prime}\right)} \\
= & 2 D_{n}(x) \frac{\left(x-x_{0}\right)\left(x-x_{1}\right) \cdots\left(x-x_{n-2}\right)}{\left(x-x_{0}^{\prime}\right)\left(x-x_{1}^{\prime}\right) \cdots\left(x-x_{n}^{\prime}\right)}=2 D_{n}(x) \frac{\mathcal{X}_{n-1}(x)}{\left(x-x_{0}^{\prime}\right)\left(x-x_{n}^{\prime}\right)},
\end{aligned}
$$

where $D_{n}$ is a polynomial of degree 2 . 
Interesting values are found at the same point as in (6):

$$
\begin{aligned}
& D_{n}\left(x_{-1}\right)=-\frac{C_{n} X_{2}\left(x_{-1}\right)\left(y_{0}-y_{-1}\right)}{2}, \\
& D_{n}\left(x_{n-1}\right)=\frac{C_{n} X_{2}\left(x_{n-1}\right)\left(y_{n}-y_{n-1}\right)}{2}, \\
& D_{n}\left(x_{0}^{\prime}\right)=\frac{C_{n} X_{2}\left(x_{0}^{\prime}\right)\left(y_{1}^{\prime}-y_{0}^{\prime}\right)}{2}, \\
& D_{n}\left(x_{n}^{\prime}\right)=-\frac{C_{n} X_{2}\left(x_{n}^{\prime}\right)\left(y_{n+1}^{\prime}-y_{n}^{\prime}\right)}{2},
\end{aligned}
$$

when $n>0$. Of course, $D_{0}=1$.

\section{Elliptic hypergeometric expansions}

Let us consider expansions of the form

$$
\sum_{k=0}^{\infty} \prod_{j}\left(z_{0}^{(j)}\right)^{ \pm 1}\left(z_{1}^{(j)}\right)^{ \pm 1} \cdots\left(z_{k}^{(j)}\right)^{ \pm 1}
$$

where $z_{k}^{(j)}$ is a combination $a_{j} x_{k}^{(j)}+b_{j}$ or $a_{j} y_{k}^{(j)}+b_{j},\left\{\ldots\left(x_{k}^{(j)}, y_{k}^{(j)}\right),\left(x_{k}^{(j)}, y_{k+1}^{(j)}\right), \ldots\right\}$ being elliptic lattices, or grids, related to a biquadratic curve (3), the same curve for each $j$.

We certainly recover at least a special case of current elliptic hypergeometric expansions, as introduced in $[4,5,30,32,34]$.

\subsection{Rational interpolatory elliptic expansions}

Rational interpolants of some function $f$ at $y_{0}, y_{1}, \ldots$, with poles at $y_{1}^{\prime}, y_{2}^{\prime}, \ldots$, are successive sums

$$
\begin{aligned}
& c_{0}=f\left(y_{0}\right), \quad c_{0}+c_{1} \frac{x-y_{0}}{x-y_{1}^{\prime}}, \quad c_{0}+c_{1} \frac{x-y_{0}}{x-y_{1}^{\prime}}+c_{2} \frac{\left(x-y_{0}\right)\left(x-y_{1}\right)}{\left(x-y_{1}^{\prime}\right)\left(x-y_{2}^{\prime}\right)}, \quad \ldots, \\
& \sum_{k=0}^{\infty} c_{k} \mathcal{Y}_{k}(x) .
\end{aligned}
$$

If, by chance, $c_{k}$ shows a similar form of ratio of products, we see special cases of hypergeometric expansions! This will happen when one expands solutions of difference equations which are simple enough. Putting the expansion in the difference equation results in recurrence relations for $c_{k}$, and we look for cases when this recurrence relation only involves two terms $c_{k}$ and $c_{k+1}$.

\section{$2.2 \quad$ Linear $1^{\text {st }}$ order difference equations}

$$
a(x)(\mathcal{D} f)(x)=c(x)(\mathcal{M} f)(x)+d(x)
$$

Where is $b$ ? The full flexibility of first order difference equations is achieved with the Riccati form [24]

$$
a(x)(\mathcal{D} f)(x)=b(x) f(\varphi(x)) f(\psi(x))+c(x)[f(\varphi(x))+f(\psi(x))]+d(x)
$$

but only linear equations will be considered here. However, (9) already allows elliptic exponentials $(c(x) \equiv a(x))$ or logarithms $(c(x) \equiv 0)$. 
We now try to expand a solution to (9) as an interpolatory series. If the initial condition is $f\left(y_{0}\right)$ at $x=x_{0}$, the difference equation allows to find

$$
f\left(y_{1}\right)=\frac{\left[a\left(x_{0}\right) /\left(y_{1}-y_{0}\right)+c\left(x_{0}\right) / 2\right] f\left(y_{0}\right)+d\left(x_{0}\right)}{a\left(x_{0}\right) /\left(y_{1}-y_{0}\right)-c\left(x_{0}\right) / 2}, \quad f\left(y_{2}\right), \quad \ldots
$$

This works fine if no division by zero is encountered. Let us call $x_{0}^{\prime}$ one of the roots of the algebraic equation

$$
\frac{a(x)}{\psi(x)-\varphi(x)}-\frac{c(x)}{2}=0, \quad \text { at } \quad x=x_{0}^{\prime}
$$

and let, as usual, $\psi\left(x_{0}^{\prime}\right)=y_{1}^{\prime}, \varphi\left(x_{0}^{\prime}\right)=y_{0}^{\prime}$. This shows that $y_{1}^{\prime}$ is a singular point of $f$, as trying to compute $f\left(y_{1}^{\prime}\right)$ from $f\left(y_{0}^{\prime}\right)$ requires a division by zero. Then $y_{2}^{\prime}, y_{3}^{\prime}, \ldots$ are poles as well. That's why the expansion in (8) starts with poles at $y_{1}^{\prime}, y_{2}^{\prime}, \ldots$ We also see that such expansions represent meromorphic functions with a natural boundary made of poles. At least, if the poles are spread on a curve, this will be discussed in Section 3.

We also manage to have the initial value $f\left(y_{0}\right)$ completely determined by the equation, i.e., independent of $f\left(y_{-1}\right)$, so, considering

$$
f\left(y_{0}\right)=\frac{\left[a\left(x_{-1}\right) /\left(y_{0}-y_{-1}\right)+c\left(x_{-1}\right) / 2\right] f\left(y_{-1}\right)+d\left(x_{-1}\right)}{a\left(x_{-1}\right) /\left(y_{0}-y_{-1}\right)-c\left(x_{-1}\right) / 2},
$$

we ask $x_{-1}$ to be a root of

$$
\frac{a(x)}{\psi(x)-\varphi(x)}+\frac{c(x)}{2}=0, \quad \text { at } \quad x=x_{-1} .
$$

Finally, we shall need the polynomials $c$ and $d$ to be of degree 3 , with $X_{2}$ as factor:

$$
c(x)=(\beta x+\gamma) X_{2}(x), \quad d(x)=(\delta x+\epsilon) X_{2}(x) .
$$

We now have enough information for understanding the

Theorem 1. The difference equation (9) on the elliptic lattice $F\left(x_{n}, y_{n}\right)=0$ of (2a)-(3), where $a, c$, and $d$ are polynomials of degree $\leqslant 3, X_{2}$ being a factor of $c$ and $d$ as in (12), has a solution with the formal expansion (8), where $x_{-1}$ is a root of (11) and $x_{0}^{\prime}$ is a root of (10), with

$$
\begin{aligned}
& c_{0}=f\left(y_{0}\right)=\frac{d\left(x_{-1}\right)}{a\left(x_{-1}\right) /\left(y_{0}-y_{-1}\right)-c\left(x_{-1}\right) / 2}=-\frac{d\left(x_{-1}\right)}{c\left(x_{-1}\right)}=-\frac{\delta x_{-1}+\epsilon}{\beta x_{-1}+\gamma}, \\
& c_{1}=\frac{\left(\delta+\beta c_{0}\right)\left(x_{0}-x_{1}^{\prime}\right)}{C_{1}\left(a\left(x_{0}\right)-c\left(x_{0}\right)\left(y_{1}-y_{0}\right) / 2\right)}=\frac{(\gamma \delta-\beta \epsilon)\left(y_{1}-y_{1}^{\prime}\right) X_{2}\left(x_{0}^{\prime}\right)}{\left(y_{1}-y_{0}^{\prime}\right)\left(x_{0}-x_{0}^{\prime}\right)\left[a\left(x_{0}\right)-c\left(x_{0}\right)\left(y_{1}-y_{0}\right) / 2\right]},
\end{aligned}
$$

and when $n \geqslant 1$,

$$
\begin{aligned}
c_{n}= & c_{1} \frac{C_{1}}{x_{1}^{\prime}-x_{0}} \frac{x_{n}^{\prime}-x_{n-1}}{C_{n}} \prod_{k=1}^{n-1} \frac{a\left(x_{k}^{\prime}\right)+c\left(x_{k}^{\prime}\right)\left(y_{k+1}^{\prime}-y_{k}^{\prime}\right) / 2}{a\left(x_{k}\right)-c\left(x_{k}\right)\left(y_{k+1}-y_{k}\right) / 2} \frac{\left(x_{k}-x_{-1}\right)\left(x_{k}-x_{0}^{\prime}\right)}{\left(x_{k}^{\prime}-x_{-1}\right)\left(x_{k}^{\prime}-x_{0}^{\prime}\right)} \\
= & -c_{1} \frac{C_{1}}{x_{1}^{\prime}-x_{0}}\left(x_{n}^{\prime}-x_{n-1}\right) \frac{\left(y_{-1}-y_{1}^{\prime}\right) \cdots\left(y_{-1}-y_{n-1}^{\prime}\right) X_{2}\left(x_{-1}\right)\left(x_{-1}-x_{0}\right) \cdots\left(x_{-1}-x_{n-2}\right)}{\left(y_{-1}-y_{1}\right) \cdots\left(y_{-1}-y_{n-2}\right)\left(x_{-1}-x_{0}^{\prime}\right) \cdots\left(x_{-1}-x_{n}^{\prime}\right)} \\
& \times \prod_{k=0}^{n-1} \frac{a\left(x_{k}^{\prime}\right)+c\left(x_{k}^{\prime}\right)\left(y_{k+1}^{\prime}-y_{k}^{\prime}\right) / 2}{a\left(x_{k}\right)-c\left(x_{k}\right)\left(y_{k+1}-y_{k}\right) / 2} \frac{\left(x_{k}-x_{-1}\right)\left(x_{k}-x_{0}^{\prime}\right)}{\left(x_{k}^{\prime}-x_{-1}\right)\left(x_{k}^{\prime}-x_{0}^{\prime}\right)} .
\end{aligned}
$$


Proof. Put the expansion (8) in

$$
\begin{aligned}
d(x) & =a(x) \mathcal{D} f(x)-c(x) \mathcal{M} f(x)=\sum_{0}^{\infty} c_{n}\left[a(x) \mathcal{D} \mathcal{Y}_{n}(x)-c(x)\left(\mathcal{M} \mathcal{Y}_{n}(x)\right]\right. \\
& =-c_{0} c(x)+\sum_{1}^{\infty} c_{n}\left[a(x) C_{n} X_{2}(x)-c(x) D_{n}(x)\right] \frac{\mathcal{X}_{n-1}(x)}{\left(x-x_{0}^{\prime}\right)\left(x-x_{n}^{\prime}\right)} .
\end{aligned}
$$

The polynomial $a(x) C_{n} X_{2}(x)-c(x) D_{n}(x)=\left[a(x) C_{n}-(\beta x+\gamma) D_{n}(x)\right] X_{2}(x)$ already has $X_{2}$ as factor from (12). A factor of degree $\leqslant 3$ remains. Complete factoring follows:

at $x_{-1}$, from (7a) and (11),

$$
a(x) C_{n} X_{2}(x)-c(x) D_{n}(x)=C_{n} X_{2}\left(x_{-1}\right)\left[a\left(x_{-1}\right)+\left(y_{0}-y_{-1}\right) c\left(x_{-1}\right) / 2\right]=0 ;
$$

at $x_{0}^{\prime}$, from $(7 \mathrm{c})$ and $(10)$,

$$
a(x) C_{n} X_{2}(x)-c(x) D_{n}(x)=C_{n} X_{2}\left(x_{0}^{\prime}\right)\left[a\left(x_{0}^{\prime}\right)-\left(y_{1}^{\prime}-y_{0}^{\prime}\right) c\left(x_{0}^{\prime}\right) / 2\right]=0 .
$$

Therefore we have three factors of first degree

$$
a(x) C_{n} X_{2}(x)-c(x) D_{n}(x)=X_{2}(x)\left(x-x_{-1}\right)\left(x-x_{0}^{\prime}\right)\left[\xi_{n}\left(x-x_{n-1}\right)+\eta_{n}\left(x-x_{n}^{\prime}\right)\right],
$$

where from $(7 d)$

$$
\xi_{n}=\frac{a\left(x_{n}^{\prime}\right) C_{n} X_{2}\left(x_{n}^{\prime}\right)-c\left(x_{n}^{\prime}\right) D_{n}\left(x_{n}^{\prime}\right)}{X_{2}\left(x_{n}^{\prime}\right)\left(x_{n}^{\prime}-x_{-1}\right)\left(x_{n}^{\prime}-x_{0}^{\prime}\right)\left(x_{n}^{\prime}-x_{n-1}\right)}=C_{n} \frac{a\left(x_{n}^{\prime}\right)+c\left(x_{n}^{\prime}\right)\left(y_{n+1}^{\prime}-y_{n}^{\prime}\right) / 2}{\left(x_{n}^{\prime}-x_{-1}\right)\left(x_{n}^{\prime}-x_{0}^{\prime}\right)\left(x_{n}^{\prime}-x_{n-1}\right)},
$$

and from $(7 \mathrm{~b})$

$$
\begin{aligned}
\eta_{n} & =\frac{a\left(x_{n-1}\right) C_{n} X_{2}\left(x_{n-1}\right)-c\left(x_{n-1}\right) D_{n}\left(x_{n-1}\right)}{X_{2}\left(x_{n-1}\right)\left(x_{n-1}-x_{-1}\right)\left(x_{n-1}-x_{0}^{\prime}\right)\left(x_{n-1}-x_{n}^{\prime}\right)} \\
& =C_{n} \frac{a\left(x_{n-1}\right)-c\left(x_{n-1}\right)\left(y_{n}-y_{n-1}\right) / 2}{\left(x_{n-1}-x_{-1}\right)\left(x_{n-1}-x_{0}^{\prime}\right)\left(x_{n-1}-x_{n}^{\prime}\right)} .
\end{aligned}
$$

Next,

$$
\begin{aligned}
0= & a(x) \mathcal{D} f(x)-c(x) \mathcal{M} f(x)-d(x) \\
= & -c_{0} c(x)-d(x)+\sum_{1}^{\infty} c_{n}\left[a(x) C_{n} X_{2}(x)-c(x) D_{n}(x)\right] \frac{\mathcal{X}_{n-1}(x)}{\left(x-x_{0}^{\prime}\right)\left(x-x_{n}^{\prime}\right)} \\
= & -c_{0} c(x)-d(x) \\
& +\sum_{1}^{\infty} c_{n} X_{2}(x)\left[\xi_{n}\left(x-x_{n-1}\right)+\eta_{n}\left(x-x_{n}^{\prime}\right)\right] \frac{\left(x-x_{-1}\right)\left(x-x_{0}\right) \cdots\left(x-x_{n-2}\right)}{\left(x-x_{1}^{\prime}\right) \cdots\left(x-x_{n}^{\prime}\right)} \\
= & -c_{0} c(x)-d(x)+X_{2}(x) \sum_{1}^{\infty} c_{n} \xi_{n} \frac{\left(x-x_{-1}\right)\left(x-x_{0}\right) \cdots\left(x-x_{n-2}\right)\left(x-x_{n-1}\right)}{\left(x-x_{1}^{\prime}\right) \cdots\left(x-x_{n}^{\prime}\right)} \\
& +X_{2}(x) \sum_{1}^{\infty} c_{n} \eta_{n} \frac{\left(x-x x_{-1}\right)\left(x-x_{0}\right) \cdots\left(x-x_{n-2}\right)}{\left(x-x_{1}^{\prime}\right) \cdots\left(x-x_{n-1}^{\prime}\right)} \\
= & -c_{0} c(x)-d(x)+c_{1} X_{2}(x) \eta_{1}\left(x-x_{-1}\right) \\
& +X_{2}(x) \sum_{1}^{\infty}\left(c_{n} \xi_{n}+c_{n+1} \eta_{n+1}\right) \frac{\left(x-x_{-1}\right)\left(x-x_{0}\right) \cdots\left(x-x_{n-2}\right)\left(x-x_{n-1}\right)}{\left(x-x_{1}^{\prime}\right) \cdots\left(x-x_{n}^{\prime}\right)}
\end{aligned}
$$




$$
=\left(x-x_{-1}\right) X_{2}(x)\left[-c_{0} \beta-\delta+c_{1} \eta_{1}+\sum_{1}^{\infty}\left(c_{n} \xi_{n}+c_{n+1} \eta_{n+1}\right) \mathcal{X}_{n}(x)\right] .
$$

$X_{2}$ is a factor everywhere, from (12), so

$$
\begin{aligned}
0= & -c_{0}(\beta x+\gamma)-(\delta x+\epsilon)+c_{1} C_{1} \frac{a\left(x_{0}\right)-c\left(x_{0}\right)\left(y_{1}-y_{0}\right) / 2}{x_{0}-x_{1}^{\prime}}\left(x-x_{-1}\right) \\
& +\sum_{1}^{\infty}\left(c_{n} \xi_{n}+c_{n+1} \eta_{n+1}\right) \mathcal{X}_{n}(x), \\
c_{0}= & f\left(y_{0}\right)=\frac{d\left(x_{-1}\right)}{a\left(x_{-1}\right) /\left(y_{0}-y_{-1}\right)-c\left(x_{-1}\right) / 2}=-\frac{d\left(x_{-1}\right)}{c\left(x_{-1}\right)}=-\left(\delta x_{-1}+\epsilon\right) /\left(\beta x_{-1}+\gamma\right), \\
c_{1}= & \frac{\left(\delta+\beta c_{0}\right)\left(x_{0}-x_{1}^{\prime}\right)}{C_{1}\left(a\left(x_{0}\right)-c\left(x_{0}\right)\left(y_{1}-y_{0}\right) / 2\right)}=\frac{(\gamma \delta-\beta \epsilon)\left(y_{1}-y_{1}^{\prime}\right) X_{2}\left(x_{0}^{\prime}\right)}{\left(y_{1}-y_{0}^{\prime}\right)\left(x_{0}-x_{0}^{\prime}\right)\left(a\left(x_{0}\right)-c\left(x_{0}\right)\left(y_{1}-y_{0}\right) / 2\right)},
\end{aligned}
$$

as

$$
\begin{aligned}
& \frac{c_{n+1}}{c_{n}}=-\frac{\xi_{n}}{\eta_{n+1}}=-\frac{C_{n}}{C_{n+1}} \frac{a\left(x_{n}^{\prime}\right)+c\left(x_{n}^{\prime}\right)\left(y_{n+1}^{\prime}-y_{n}^{\prime}\right) / 2}{a\left(x_{n}\right)-c\left(x_{n}\right)\left(y_{n+1}-y_{n}\right) / 2} \frac{\left(x_{n}-x_{-1}\right)\left(x_{n}-x_{0}^{\prime}\right)\left(x_{n}-x_{n+1}^{\prime}\right)}{\left(x_{n}^{\prime}-x_{-1}\right)\left(x_{n}^{\prime}-x_{0}^{\prime}\right)\left(x_{n}^{\prime}-x_{n-1}\right)}, \\
& c_{n}=\cdots \frac{\left.x_{n}^{\prime}-x_{n-1}\right)}{C_{n}} \prod^{n-1} \frac{a\left(x_{k}^{\prime}\right)+c\left(x_{k}^{\prime}\right)\left(y_{k+1}^{\prime}-y_{k}^{\prime}\right) / 2}{a\left(x_{k}\right)+c\left(x_{k}\right)\left(y_{k+1}-y_{k}\right) / 2} \mathcal{X}_{n}\left(x_{-1}\right) \mathcal{X}_{n}\left(x_{0}^{\prime}\right) .
\end{aligned}
$$

The formula (13) achieves a construction of hypergeometric type, as each term is a product of values of elliptic functions with arguments in arithmetic progression. The exact order of each term, i.e., the number of zeros and poles in a minimal parallelogram, is not obvious [33]. Of course, a factor like, say, $x_{-1}-x_{k}$ is an elliptic function of order 2 of $t_{0}+k h$ from (4). The same order holds for the ratio

$$
\frac{x_{-1}-x_{k}}{y_{-1}-y_{k}}=\frac{x_{-1}-\mathcal{E}_{1}\left(t_{0}+k h\right)}{y_{-1}-\mathcal{E}_{2}\left(t_{0}+k h\right)},
$$

as zeros of the numerator and the denominator cancel each other.

Similar effects probably hold in other ratios encountered in (13), such as

$$
\frac{a\left(x_{k}\right)-c\left(x_{k}\right)\left(y_{k+1}-y_{k}\right) / 2}{\left(x_{k}-x_{-1}\right)\left(x_{k}-x_{0}^{\prime}\right)}
$$

but it is not clear if more can be obtained by keeping elementary means, or if more elliptic function machinery (theta functions) is needed. An elementary description holds however in the "logarithmic" case $c(x) \equiv 0$. Then, (10) and (11) already tell that $x_{-1}$ and $x_{0}^{\prime}$ are two roots of $a(x)=0$. And as the polynomial $a$ has degree 3 in Theorem 1 , let $a(x)=\left(x-x_{-1}\right)\left(x-x_{0}^{\prime}\right)(x-\zeta)$. Then, from (13),

$$
\begin{aligned}
c_{n}=- & c_{1} \frac{C_{1}}{x_{1}^{\prime}-x_{0}}\left(x_{n}^{\prime}-x_{n-1}\right) \frac{\left(y_{-1}-y_{1}^{\prime}\right) \cdots\left(y_{-1}-y_{n-1}^{\prime}\right) X_{2}\left(x_{-1}\right)\left(x_{-1}-x_{0}\right) \cdots\left(x_{-1}-x_{n-2}\right)}{\left(y_{-1}-y_{1}\right) \cdots\left(y_{-1}-y_{n-2}\right)\left(x_{-1}-x_{0}^{\prime}\right) \cdots\left(x_{-1}-x_{n}^{\prime}\right)} \\
& \times \prod_{k=0}^{n-1} \frac{a\left(x_{k}^{\prime}\right)}{a\left(x_{k}\right)} \frac{\left(x_{k}-x_{-1}\right)\left(x_{k}-x_{0}^{\prime}\right)}{\left(x_{k}^{\prime}-x_{-1}\right)\left(x_{k}^{\prime}-x_{0}^{\prime}\right)}, \\
c_{n} \mathcal{Y}_{n}(x)= & -c_{1} \frac{C_{1}}{x_{1}^{\prime}-x_{0}}\left(x_{n}^{\prime}-x_{n-1}\right) \\
& \times \frac{\left(y_{-1}-y_{1}^{\prime}\right) \cdots\left(y_{-1}-y_{n-1}^{\prime}\right) X_{2}\left(x_{-1}\right)\left(x_{-1}-x_{0}\right) \cdots\left(x_{-1}-x_{n-2}\right)}{\left(y_{-1}-y_{1}\right) \cdots\left(y_{-1}-y_{n-2}\right)\left(x_{-1}-x_{0}^{\prime}\right) \cdots\left(x_{-1}-x_{n}^{\prime}\right)} \\
& \times \prod_{k=0}^{n-1} \frac{\left(x_{k}^{\prime}-\zeta\right)\left(x-y_{k}\right)}{\left(x_{k}-\zeta\right)\left(x-y_{k+1}^{\prime}\right)} .
\end{aligned}
$$




\section{A word on convergence}

\subsection{Average behaviour}

We expect products occurring in (13) or (14) to behave like powers, like

$$
\prod_{1}^{n}\left(x-x_{k}\right)=\prod_{1}^{n}\left(x-\mathcal{E}\left(t_{0}+k h\right)\right) \approx \Phi_{+}(x)^{n} .
$$

What is $\Phi_{+}(x)=\exp \mathcal{V}_{+}(x)$, where $\mathcal{V}_{+}$is the complex potential of the distributions of $x_{k}$ ? For $x_{k}^{\prime}$, we write $\mathcal{V}_{-}(x)$. For $y$, let us use the symbol $\mathcal{W}$.

The main behaviour of the $n^{\text {th }}$ term of (14) is therefore

$$
\begin{aligned}
\exp ( & n\left(\mathcal{W}_{-}\left(y_{-1}\right)-\mathcal{W}_{+}\left(y_{-1}\right)+\mathcal{V}_{+}\left(x_{-1}\right)-\mathcal{V}_{-}\left(x_{-1}\right)+\mathcal{V}_{-}(\zeta)\right. \\
& \left.\left.-\mathcal{V}_{+}(\zeta)+\mathcal{W}_{+}(x)-\mathcal{W}_{-}(x)\right)\right)
\end{aligned}
$$

Remark that we will only need $\mathcal{V}=\mathcal{V}_{+}-\mathcal{V}_{-}$and $\mathcal{W}=\mathcal{W}_{+}-\mathcal{W}_{-}$.

If $h$ is a general complex number, $x_{k}$ fill the whole complex plane and no convergence occurs.

Let $h$ be a real irrational multiple of a period $\omega$, then the same factors reappear approximately in the product after $N$ steps if $N h$ is close to an integer times $\omega . \Phi(x)$ is the limit of the $N^{\text {th }}$ roots of such products. The various $k h$, for $k=1,2, \ldots, N$, modulo $\omega$, fill uniformly the segment $[0, \omega]$, and $x_{k}$ fill a curve which is the set of $\mathcal{E}\left(t_{0}+u\right), u \in[0, \omega]$ : for any $j$ in $\{1,2, \ldots, N\}$, there is a $k$ such that $k h$ is close to $j \omega / N$ modulo $\omega$. Indeed, let $N h$ be close to $M_{N} \omega$, with $\operatorname{gcd}\left(N, M_{N}\right)=1$. Then,

$$
k h-\frac{j \omega}{N}=\omega\left(\frac{h}{\omega}-\frac{M_{N}}{N}\right) k+\omega \frac{k M_{N}-j}{N},
$$

to any $j$, there are integers $k$ and $m$ such that $k M_{N}-m N=j$ (Bezout).

So, we rearrange the product as

$$
\Phi(x) \sim\left[\prod_{j=1}^{N}\left(x-\mathcal{E}\left(j \omega / N+t_{0}\right)\right)\right]^{1 / N} \sim \exp \left[\frac{1}{\omega} \int_{0}^{\omega} \log \left(x-\mathcal{E}\left(u+t_{0}\right)\right) d u\right] .
$$

As $\mathcal{E}$ is the inversion of an elliptic integral of the first kind,

$$
u+t_{0}=\int^{\mathcal{E}} \frac{d v}{\sqrt{P(v)}},
$$

we have

$$
\Phi(x)=\exp \left[\frac{1}{\omega} \int_{\left\{x_{n}\right\}} \frac{\log (x-v) d v}{\sqrt{P(v)}}\right],
$$

where $\left\{x_{n}\right\}$ is the locus $=\left\{\mathcal{E}\left(u+t_{0}\right)\right\}, u \in[0, \omega]$. The constant $1 / \omega$ is such that $\Phi(x) \sim x$ for large $x$ :

$$
\omega=\int_{\left\{x_{n}\right\}} \frac{d v}{\sqrt{P(v)}}
$$

So, let the complex potential

$$
\mathcal{V}_{+}(x)=\frac{1}{\omega} \int_{\left\{x_{n}\right\}} \frac{\log (x-v) d v}{\sqrt{P(v)}}
$$

$\left(\mathcal{V}_{-}\right.$will be used with $x_{n}^{\prime}$, and $\mathcal{W}_{ \pm}$for $y_{n}$ and $\left.y_{n}^{\prime}\right)$. 
The formula for the potential will be linear after a convenient conformal map.

One has the derivative

$$
\mathcal{V}_{+}^{\prime}(x)=\frac{1}{\omega} \int_{\left\{x_{n}\right\}} \frac{d v}{(x-v) \sqrt{P(v)}}
$$

with $\xi$ such that $x=\mathcal{E}(\xi), d x / d \xi=\sqrt{P(x)}$.

So, $\mathcal{V}_{+}^{\prime}(x)$ and $\mathcal{V}_{-}^{\prime}(x)$ are contour integrals on the locii filled by $\left\{x_{n}\right\}$ and $\left\{x_{n}^{\prime}\right\}$ drawn by $\mathcal{E}\left(n h+t_{0}\right)$ and $\mathcal{E}\left(n h+t_{0}^{\prime}\right)$. If $x$ is between these two locii, the two contour integrals of $\frac{d v}{(x-v) \sqrt{P(v)}}$ are the same for $\mathcal{V}_{+}^{\prime}(x)$ and $\mathcal{V}_{-}^{\prime}(x)$, up to the residue at $v=x$ :

$$
\mathcal{V}^{\prime}(x)=\mathcal{V}_{+}^{\prime}(x)-\mathcal{V}_{-}^{\prime}(x)=\frac{2 \pi i}{\omega \sqrt{P(x)}} \Rightarrow \frac{d \mathcal{V}(x)}{d \xi}=\frac{2 \pi i}{\omega}
$$

We see that the real part of $\mathcal{V}$ remains constant on lines in the $\xi$-plane such that $d \xi / \omega$ is real, i.e., on parallel lines sharing the $\omega$-direction.

Remember that the step $h$ has been supposed to be a real multiple of $\omega$, so the arguments in arithmetic progression of step $h$ in the $\xi$-plane of the elliptic functions defining a sequence $x_{n}$, or $y_{n}$, etc. happen to draw parallel lines with the $\omega$-direction! The real part of $\mathcal{V}(\zeta)-\mathcal{V}\left(x_{-1}\right)$ occurring in (15) is therefore $2 \pi /|\omega|$ times the distance between, say, $\xi_{\zeta}$, if $\zeta$ is the value of the elliptic function at $\xi_{\zeta}$, and the line leading to the $\left\{x_{n}\right\}$ sequence.

The remaining terms of (15) lead to a convergence behaviour dominated by

$$
\exp \left(-n \operatorname{Im} 2 \pi\left(\xi_{x}-\xi_{\zeta}\right) / \omega\right)
$$

where $\xi_{x}$ is sent to $x$ by the elliptic function.

Of course, convergence holds while $x$ is between the locus of $x_{n}$ and the corresponding locus (equipotential line) containing $\zeta$.

In a Jacobian setting, evaluation of (16) typically involves $\exp \left(-n \pi K^{\prime} / K\right)$, well known in Zolotarev problems solutions and generalizations [8].

Rate of approximation has already been related to potential problems by Walsh [36, chapter 9], in papers and books going back to the 1930s! See also Ganelius [7]. For more recent surveys and papers, the works of Bagby [3], and by Gončar and colleagues are recommended $[8,9,10,11,12]$.

It is quite remarkable that configurations of particles in statistical physics $[18,19,20]$ are described in the same way than zeros and poles of rational approximations $[3,8,9,10,11,12$, $25,29,35]$.

\subsection{Exceptional cases}

The properties of the irrational number relating the step $h$ to a period $\omega$ must also be considered [31]. Indeed, (14) contains a division by a factor $\left(y_{-1}-y_{n-2}\right)$ which is the difference of the values of a function of period $\omega$ at arguments differing by an integer multiple of $h$, so that the result will be small whenever $(n-1) h$ is close to an integer multiple of $\omega$. The difference will never vanish, as $h / \omega$ is irrational, but could become VERY small infinitely often. The set of irrational $h / \omega$ that could destroy the convergence estimate above is fortunately of vanishing measure in the set of real numbers, as shown by Hardy and Littlewood in [14] (and reproduced by Lubinsky in [21, pp. 854-855 and 871]). 


\section{Acknowledgments}

Many thanks to the organizers of the workshop "Elliptic Integrable Systems, Isomonodromy Problems, and Hypergeometric Functions" (Hausdorff Center for Mathematics, Bonn, July 2008), to A. Aptekarev, B. Beckermann, A.C. Matos, F. Wielonsky, of the Laboratoire Paul Painlevé UMR 8524, Université de Lille 1, France, who organized their $3^{\text {èmes }}$ Journées Approximation on May 15-16, 2008. Many thanks too to R. Askey, L. Haine, M. Ismail, F. Nijhoff, A. Ronveaux, and, of course, V. Spiridonov and A. Zhedanov for their preprints, interest, remarks, and kind words. Many thanks to the referees for expert and careful reading, and kind words too.

This paper presents research results of the Belgian Programme on Interuniversity Attraction Poles, initiated by the Belgian Federal Science Policy Office.

\section{References}

[1] Andrews G.E., Askey R., Roy R., Special functions, Encyclopedia of Mathematics and its Applications, Vol. 71, Cambridge University Press, Cambridge, 1999.

[2] Askey R., Wilson J., Some basic hypergeometric orthogonal polynomials that generalize Jacobi polynomials, Mem. Amer. Math. Soc. 54 (1985), no. 319.

[3] Bagby T., On interpolation by rational functions, Duke Math. J. 36 (1969), 95-104.

[4] Burskii V.P., Zhedanov A.S., The Dirichlet and the Poncelet problems, RIAM Symposium No.16ME-S1 "Physics and Mathematical Structures of Nonlinear Waves" (November 15-17, 2004, Kyushu University, Kasuga, Fukuoka, Japan), 2004, 22-26, available at http://www.riam.kyushu-u.ac.jp/fluid/meeting/16ME-S1/papers/Article_No_24.pdf.

[5] Burskii V.P., Zhedanov A.S., Dirichlet and Neumann Problems for string equation, Poncelet problem and Pell-Abel equation, SIGMA 2 (2006), 041, 5 pages, math.AP/0604278.

[6] Burskii V.P., Zhedanov A.S., On Dirichlet, Poncelet and Abel problems, arXiv:0903.2531.

[7] Ganelius T., Degree of rational approximation, in Lectures on Approximation and Value Distribution, Editors T. Ganelius et al., Sém. Math. Sup., Vol. 79, Presses Univ. Montréal, Montreal, Que., 1982, 9-78.

[8] Gončar A.A., The problems of E. I. Zolotarev which are connected with rational functions, Mat. Sb. 78 (120) (1969), 640-654 (English transl.: Math. USSR-Sb. 7 (1969), 623-635).

[9] Gonchar A.A., Rational approximations of analytic functions, Sovrem. Probl. Mat. Current Problems in Mathematics, no. 1, Ross. Akad. Nauk, Inst. Mat. im. V.A. Steklova, Moscow, 2003, 83-106 (in Russian).

[10] Gonchar A.A., Rakhmanov E.A., Equilibrium distributions and the rate of rational approximation of analytic functions, Mat. Sb. 134 (176) (1987), 306-352 (English transl.: Math. USSR-Sb. 62 (1989), 305-348).

[11] Gonchar A.A., Rakhmanov E.A., Suetin S.P., On the rate of convergence of Padé approximants of orthogonal expansions, in Progress in Approximation Theory (Tampa, FL, 1990), Springer Ser. Comput. Math., Vol. 19, Springer, New York, 1992, 169-190.

[12] Gonchar A.A., Suetin S.P., On Padé approximants of meromorphic functions of Markov type, Current Problems in Mathematics, no. 5, Ross. Akad. Nauk, Inst. Mat. im. V.A. Steklova, Moscow, 2004, 68 pages (in Russian), available at http://www.mi.ras.ru/spm/pdf/005.pdf.

[13] Grünbaum F.A., Haine L., On a $q$-analogue of Gauss equation and some $q$-Riccati equations, in Special Functions, q-Series and Related Topics (Toronto, ON, 1995), Editors M.E.H. Ismail et al., Fields Inst. Commun., Vol. 14, Amer. Math. Soc., Providence, RI, 1997, 77-81.

[14] Hardy G.H., Littlewood J.E., Notes on the theory of series. XXIV. A curious power-series, Proc. Cambridge Philos. Soc. 42 (1946), 85-90.

[15] Ismail M.E.H., Classical and quantum orthogonal polynomials in one variable, with two chapters by Walter Van Assche, Encyclopedia of Mathematics and its Applications, Vol. 98, Cambridge University Press, Cambridge, 2005.

[16] Koekoek R., Swarttouw R.F., The Askey-scheme of hypergeometric orthogonal polynomials and its $q-$ analogue, Delft University of Technology, Faculty of Information Technology and Systems, Department of Technical Mathematics and Informatics, Report no. 98-17, 1998, math.CA/9602214. 
[17] Koornwinder T.H., Compact quantum groups and $q$-special functions, in Representations of Lie Groups and Quantum Groups (Trento, 1993), Editors V. Baldoni and M.A. Picardello, Pitman Res. Notes Math. Ser., Vol. 311, Longman Sci. Tech., Harlow, 1994, 46-128, Chapters 1, 2: General compact quantum groups, a tutorial, hep-th/9401114, Chapters 3, 4: q-special functions, a tutorial, math.CA/9403216.

[18] Loutsenko I., Spiridonov V., Spectral self-similarity, one-dimensional Ising chains and random matrices, Nuclear Phys. B $\mathbf{5 3 8}$ (1999), 731-758.

[19] Loutsenko I., Spiridonov V., Soliton solutions of integrable hierarchies and Coulomb plasmas, J. Statist. Phys. 99 (2000), 751-767, cond-mat/9909308.

[20] Loutsenko I., Spiridonov V., A critical phenomenon in solitonic Ising chains, SIGMA 3 (2007), 059, 11 pages, arXiv:0704.3173.

[21] Lubinsky D.S., Rogers-Ramanujan and the Baker-Gammel-Wills (Padé) conjecture, Ann. of Math. (2) 157 (2003), 847-889, math.CA/0402305.

[22] Magnus A.P., Associated Askey-Wilson polynomials as Laguerre-Hahn orthogonal polynomials, in Orthogonal Polynomials and their Applications (Segovia, 1986), Editors M. Alfaro et al., Lecture Notes in Math., Vol. 1329, Springer, Berlin, 1988, 261-278.

[23] Magnus A.P., Special non uniform lattice (snul) orthogonal polynomials on discrete dense sets of points, J. Comp. Appl. Math. 65 (1995), 253-265, math.CA/9502228.

[24] Magnus A.P., Rational interpolation to solutions of Riccati difference equations on elliptic lattices, J. Comp. Appl. Math., 2009, to appear, http://dx.doi.org/10.1016/j.cam.2009.02.047, preprint available at http://perso.uclouvain.be/alphonse .magnus/num3/MagnusLuminy2007.pdf.

[25] Meinguet J., An electrostatic approach to the determination of extremal measures, Math. Phys. Anal. Geom. 3 (2000), 323-337.

[26] Milne-Thomson L.M., The calculus of finite differences, Macmillan and Co., Ltd., London, 1951, available at http://www.archive.org/details/calculusoffinite032017mbp.

[27] Nikiforov A.F., Suslov S.K., Classical orthogonal polynomials of a discrete variable on nonuniform lattices, Lett. Math. Phys. 11 (1986), 27-34.

[28] Nikiforov A.F., Suslov S.K., Uvarov V.B., Classical orthogonal polynomials of a discrete variable, Series in Computational Physics, Springer-Verlag, Berlin, 1991.

[29] Saff E.B., Totik V., Logarithmic potentials with external fields. Appendix B by Thomas Bloom, Grundlehren der Mathematischen Wissenschaften, Vol. 316, Springer-Verlag, Berlin, 1997.

[30] Spiridonov V.P., Essays on the theory of elliptic hypergeometric functions, Uspekhi Mat. Nauk 63 (2008), no. 3, 3-72 (English transl.: Russ. Math. Surv. 63 (2008), 405-472), arXiv:0805.3135

[31] Spiridonov V.P., On the analytical properties of infinite elliptic hypergeometric series, talk presented at the workshop "Elliptic Integrable Systems, Isomonodromy Problems, and Hypergeometric Functions", Hausdorff Center for Mathematics, Bonn, July 2008.

[32] Spiridonov V.P., Zhedanov A.S., Generalized eigenvalue problem and a new family of rational functions biorthogonal on elliptic grids, in Special Functions 2000: Current Perspective and Future Directions (Tempe, AZ, 2000), Editors J. Bustoz et al., NATO Sci. Ser. II, Math. Phys. Chem., Vol. 30, Kluwer Acad. Publ., Dordrecht, 2001, 365-388.

[33] Spiridonov V.P., Zhedanov A.S., Private communication to the author, Wednesday, July 23, 2008, on the Rhine river, en route towards Königswinter.

[34] Spiridonov V.P., Zhedanov A.S., Elliptic grids, rational functions, and the Padé interpolation Ramanujan J. 13 (2007), 285-310.

[35] Stahl H., Convergence of rational interpolants, in Numerical Analysis, (Louvain-la-Neuve, 1995), Bull. Belg. Math. Soc. Simon Stevin 1996 (1996), suppl., 11-32.

[36] Walsh J.L., Interpolation and approximation by rational functions in the complex domain, 4th ed., American Mathematical Society Colloquium Publications, Vol. 20, American Mathematical Society, Providence, R.I., 1965. 